Meeting report

\title{
Update on endocrine aspects of breast cancer: Report from the 23rd San Antonio Breast Cancer Symposium, San Antonio, Texas, USA, 6-9 December 2000
}

Stephen RD Johnston

Department of Medicine, Royal Marsden Hospital and Institute of Cancer Research, London, UK

Correspondence: Dr Stephen RD Johnston, MA, MRCP, PhD, Senior Lecturer and Consultant Medical Oncologist, Department of Medicine, Royal Marsden Hospital and Institute of Cancer Research, Fulham Road, London, SW3 6JJ, UK. Tel: +44 02078082748 ; fax: +44 020 7352 5441; e-mail: stephen@icr.ac.uk

Received: 12 January 2001

Accepted: 19 January 2001

Published: 26 March 2001
Breast Cancer Res 2001, 3:180-182

(C) 2001 BioMed Central Ltd

(Print ISSN 1465-5411; Online ISSN 1465-542X)

\section{Introduction}

As in previous years, this has become one of the key meetings of the year for both scientists and clinicians who are involved in breast cancer, and in particular provides a forum in which to discuss the more translational aspects of current research. This year was no exception, and there was much enthusiasm among those interested in endocrine aspects about new clinical and preclinical data regarding several novel agents, including pure antioestrogens, aromatase inhibitors and selective oestrogen receptor modulators (SERMs), as well as continuing studies to elucidate the molecular biology of oestrogen receptor (ER) function.

\section{Pure antioestrogens for tamoxifen-resistant advanced breast cancer}

The fact that clinical responses are seen with second-line endocrine therapy in ER-positive breast cancer after the development of resistance to tamoxifen implies that some tumours retain partial hormone sensitivity. Several years ago, considerable interest was generated by a small phase II study that suggested substantial activity in tamoxifen-resistant breast cancer with the pure steroidal antioestrogen ICl 182,780, which was devoid of any intrinsic agonist activity. This observation was supported by experimental data in MCF-7 'tamoxifen-resistant' xenografts, which confirmed that ICI 182,780, but not the structurally related triphenylethylene toremiphene, could inhibit the growth of resistant tumours.
These observations prompted two large randomized phase III trials to be initiated in women with advanced tamoxifen-resistant breast cancer that compared $\mathrm{ICl}$ 182,780 (Faslodex; AstraZeneca, Macclesfield, UK) with anastrozole, and the first data from these trials were reported at the San Antonio meeting. Both trials demonstrated that the clinical efficacy of Faslodex was identical to that of anastrozole in tamoxifen-resistant advanced breast cancer with respect to the primary end-point, namely time to disease progression. In the European trial reported by Howell (Christie Hospital, Manchester, UK) the response rate to Faslodex was $20.7 \%$ versus $15.7 \%$ for anastrozole, with an additional 23.9 and 29.3\% patients, respectively, deriving clinical benefit as determined by stable disease for at least 6 months. In the American study reported by Osborne (Baylor College of Medicine, Houston, TX, USA), which unlike the European study was a double-blind design with a slightly greater number of patients who were known to be ER-positive, the response rates were $17.5 \%$ for both arms. Interestingly, there was a nonsignificant trend towards a longer duration of response for Faslodex (median 19.3 versus 10.5 months), and an additional 24.8 and $18.6 \%$ of patients, respectively, had stable disease for 6 months. The toxicity profiles were similar in terms of endocrine effects, and in the double-blind US study the incidence of injection site reactions was identical in the Faslodex and placebo arms of the trial. 
These data therefore confirm that a pure steroidal antioestrogen is active in tamoxifen-resistant breast cancer, and in these two randomized trials is at least as effective as a third-generation aromatase inhibitor, a drug class that has become the standard of care. The real issue is at what point in the sequence of endocrine therapy options for advanced breast cancer should a pure antiestrogen be used. With the potential for incorporation of aromatase inhibitors as first-line therapy, it will become important to determine whether pure antioestrogens are active after failure of aromatase inhibitors. Data were presented by Thurliman (SAKK, St Galen, Switzerland) that confirm that responses to tamoxifen may be still be seen after anastrozole treatment, and small phase II studies are in progress with Faslodex in this scenario to determine the optimal sequence of therapies.

\section{Aromatase inhibitors are superior to tamoxifen as first-line therapy}

Data were presented, on behalf of the International Letrozole Study Group, from the largest prospective single study of an aromatase inhibitor versus tamoxifen as firstline therapy in advanced breast cancer. Letrozole was associated not only with a higher response rate than was tamoxifen (30\% versus $20 \% ; P=0.0006)$, but also with a significantly longer time to disease progression (9.4 months versus 6 months; $P=0.0001)$. The improved likelihood of benefit was seen in all subgroups. Likewise, Paridaens (EORTC, Leuven, Belgium) presented a smaller randomized phase II first-line study conducted by the European Organization for Research in Cancer Therapy, in which the response rate to the steroidal aromatase inhibitor exemestane was substantially higher than that to tamoxifen (45\% versus 14\%). A larger randomized phase III study is underway on the basis of these promising data. Thus, there are now published data for all of the third-generation aromatase inhibitors (anastrozole, letrozole and exemestane) that indicate that these drugs may be superior to tamoxifen as first-line therapy in ER-positive postmenopausal advanced breast cancer.

The aromatase inhibitor letrozole also appears to be more effective than tamoxifen when given as 4-month preoperative (neoadjuvant) therapy to 337 postmenopausal women with ER-positive and/or progesterone receptor-positive, large operable or locally advanced breast cancer. This was manifest both as an improved response rate (55\% versus $36 \% ; P<0.001$ ) and as an increase in the ability to perform breast-conserving surgery. Ellis (Duke University, Durham, NC, USA) presented data that tumours that coexpressed HER-2 (<15\%) were found to be much more likely to respond to letrozole and tamoxifen. Hitherto it has been assumed that ER-positive, HER-2-positive breast cancers are endocrine resistant, but these interesting data imply that this may differ depending on the endocrine therapeutic approach.

\section{New selective estrogen receptor modulators}

It has already been shown that the SERM raloxifene significantly reduced the incidence of breast cancer in a postmenopausal osteoporosis population of women. In an update of this study of over 7700 women by Lippman (Lombardi Cancer Center, Georgetown, Washington, DC, USA), data were presented that indicate that the greatest risk reduction by raloxifene may be seen in those women with the higher lifetime exposure to oestrogen as indicated by surrogate end-points such as bone mineral density, body mass index and serum oestradiol levels. The updated data confirm that, unlike tamoxifen, raloxifene was not associated with any increase in risk of endometrial cancer (relative risk 0.91), although the relative risk of thrombotic events remains a concern. The current STAR chemoprevention trial comparing the effects of tamoxifen with raloxifene will hopefully characterize further those patients who derive most benefit from chemoprevention, and define further the risk-benefit for each intervention.

Preclinical data were presented on several other SERM compounds that are in early clinical development. LY 353381 (Arzoxifene; Eli Lilly, Indianapolis, IN, USA) is a benzothiophene analogue that is similar, but not identical to raloxifene. Although this agent has been shown to have antioestrogenic effects on the rat uterus, data were presented by Dardes (Northwestern University Medical School, Chicago, IL, USA) that showed that, in an endometrial cancer model in athymic mice, similar growth stimulatory effects were seen as compared with tamoxifen. In contrast, ERA-923 is a novel SERM that appears to have an improved preclinical profile as compared with tamoxifen and raloxifene. Greenberger (Wyeth-Ayerst Research, Radnor, PA, USA) presented data that not only was ERA-923 devoid of agonist effects in an immature rat uterotrophic model and failed to stimulate EnCa101 endometrial xenografts in vivo, but it also lacked any stimulatory effect and inhibited the growth of tamoxifen-resistant MCF-7 breast cancer xenografts. On the basis of these data, the drug is currently being evaluated in clinical trials in women in whom tamoxifen treatment has failed.

EM-652 is a prodrug of EM-800, a SERM that has also been investigated in tamoxifen-resistant breast cancer. Data were presented by Johnston (Royal Marsden Hospital and Institute of Cancer Research, London, UK) that demonstrated that EM-652, unlike raloxifene, is a more potent antioestrogen that is totally devoid of any agonist effects in the immature rat uterotrophic assay. Likewise, data were presented by Martel (Laval University Medical Centre, Quebec, QC, Canada) that showed that EM-652 is a nonsteroidal compound that appears to be a pure antioestrogen in human endometrial adenocarcinoma cells, and behaves differently from other SERMs such as raloxifene and lasofoxifene. Other triphenylethylene structures related to tamoxifen continue to be investigated, 
including compounds such lasofoxifene and GW-7604. Data were presented by Bentram (Northwestern University Medical School, Chicago, IL, USA) with the latter compound, which appears to have lesser agonist properties that tamoxifen in specific preclinical models. However, it remains unclear to what extent these preclinical models are in any way predictive of clinical outcome in the treatment of breast cancer.

\section{Understanding the molecular biology of oestrogen receptor}

A special minisymposium was dedicated to reviewing the latest aspects of ER structure and function. The emerging biology of ER- $\beta$, together with its contrasting effects to those of ER- $\alpha$, was discussed by Gustaffson (Karolinska Instutet, Stockholm, Sweden). Experiments with ER- $\beta$ knockout mice have demonstrated that, in the uterus, cell proliferation is markedly enhanced, implying that ER- $\beta$ may have some form of balancing role. ER- $\beta$ levels may fluctuate during physiological breast development and lactation, and ongoing studies in human breast carcinomas may determine the role of levels of ER- $\beta$ relative to those of ER$\alpha$ in determining clinical outcome to endocrine therapy.

The complexity of the structure-function relationship between ER and its various ligands was reviewed by Greene (University of Chicago, Chicago, IL, USA), and may explain some aspects of the different outcomes, which depend on which ligands interact with the receptor. After ligand binding there appear to be a multiplicity of ligand-induced surfaces that act as docking sites for coactivators and corepressors. Structural differences between ER- $\alpha$ and ER- $\beta$ may explain agonist versus antagonist responses to the same ligand, although the full interaction between the AF-1 and AF-2 transactivation sites is not yet fully understood. The possibility exists, on the basis of crystallographical modelling, that small-molecule mimetics could be synthesized to inhibit key protein-protein interactions that are crucial to liganded ER function. The molecular pharmacology of the different SERMs was reviewed by McDonnell (Duke University, Durham, NC, USA), and these effects may also be explained by different structure-function relationships of each compound with ER. For example, GW-5638, which inhibits the growth of tamoxifen-resistant tumours in oophorectomized mice, induces a different conformational shape with ER compared with tamoxifen, and as such prevents a novel coactivator $(\mathrm{C} \circ \mathrm{A} \alpha \beta \mathrm{V})$ from binding to $\mathrm{ER}$.

The concept is emerging that ER may exist in one of three active states: repressor, neutral or coactivator. As such, one factor that may dictate the response to a given ligand is the relative levels of corepressor and coactivator proteins. Experimental data suggest that overexpression of corepressors may convert all SERMs into agonists in a given cell system, thus confirming that cell context in addition to receptor isotype and ligand may determine the biological response. Likewise, mutations in critical domains of ER may alter the response. Jordan (Lurie Comprehensive Cancer Center, Northwestern University, Chicago, IL, USA) presented data with a mutated ER (D351Y) isolated from a tamoxifen-stimulated tumour line, in which the amino acid involved appeared critical for interaction with a ligand's side chain. Although ER mutations may be relatively rare, any change in structure at crucial sites may dramatically alter the biological response to individual ligands.

\section{Conclusion}

The emergence into the clinic of several novel endocrine therapies has given clinicians new options in the management of breast cancer, although the correct sequence of treatment still needs to be established. There is little doubt that, in time, tamoxifen will be replaced by more specific targeted therapies, and that the profile of the current SERMs is of considerable interest for early-stage disease and possibly prevention. At the same time, our understanding of oestrogen receptor biology and the consequence of ligand interaction in different tissues is expanding, which should allow us to maximize the properties of these various new endocrine agents which are now available. 